\title{
Performance of TPC based ranging signal for more than 2 services multiplexing
}

\author{
Seung Tae Kim ${ }^{1}$, Kap-Jin Kim², Ki-Won Song ${ }^{2}$, and Jae Min $\mathrm{Ahn}^{1, *}$ \\ ${ }^{1}$ Dept. of Radio Science and Information Communications Engineering, Chungnam National University, S. Korea \\ ${ }^{2}$ Agency for Defence Development, S. Korea
}

\begin{abstract}
This paper presents signal structure and power efficiency performance for simultaneous transmission of Global Navigation Satellite System (GNSS) service signals based on Tiered Polyphase Code (TPC). For the simultaneous transmission of three or more service signals, the intermodulation terms addition and modification of the power allocations for the signal multiplexing are applied first to the spreading signal with the binary pseudorandom noise (PRN) code and a constant envelope signal is generated. Then, ZadoffChu sequence is applied as a secondary code to generate a multiplexed satellite navigation signal having a constant envelope characteristic. Simulation results show that power efficiency performance of more than $80 \%$ can be achieved in three service signal multiplexing.
\end{abstract}

\section{Introduction}

The use of Tiered Code (TC) using pseudo random noise (PRN) code of hierarchical structure is becoming popular as a method to facilitate signal acquisition in weak signal environment of Global Navigation Satellite System (GNSS) navigation signal. TC, which consists of a dual code structure of primary and secondary codes, is a binary PRN code in which both codes are combined to increase processing gain. Tiered Polyphase Code (TPC) structures have been proposed to reduce intra-system interference as well as increase processing gain by applying non-binary polyphase codes to the secondary codes. [1,2]

On the other hand, with the proliferation of GNSS services, attempts have been made to simultaneously transmit more service signals using the same frequency band. Simultaneous transmission of three or more signals may degrade the power efficiency of the satellite high power amplifier. In order to minimize the degradation of power efficiency, various methods have been proposed to keep the envelope of the generated signal constant. [3] Most of the proposed methods are based on the assumption that the simultaneous transmission signals to be multiplexed have binary values. Therefore, when the simultaneous transmission is performed based on the TPC, the signal generation structure needs to be changed since the secondary code of the TPC is non-binary.

In this paper, we propose a signal generation structure that can maintain constant envelope characteristics when simultaneously transmitting three or more GNSS service signals based on TPC. Also, it is shown that the power efficiency of the amplifier is not different from the power efficiency of the binary signals during the amplification of the signal generated by the proposed method.

\section{Generation of the multiplexed signal}

In order to generate a transmission signal by multiplexing three or more signals based on TPC, signals to be multiplexed are defined as follows.

$$
s_{i}(t)=d_{i}(t) C_{p, i}(t) C_{s}(t), i=1, \ldots, N
$$

where $\mathrm{N}$ is the number of services to be multiplexed, $d_{i}(t)$ denotes a data signal of the i-th service, $C_{p, i}(t)$ denotes a primary code of the i-th service, $C_{s}(t)$ denotes the secondary code common to all services and is non-binary polyphase sequence.

In general, multiplexed and transmitted signals can be represented as functions of the signals for multiplexing. The signals multiplexed in the baseband are frequency shifted by the carrier signal and transmitted in the allocated frequency band. The following equation (2) shows this multiplexing process, where $f\left(s_{i}(t), i=1, \ldots, N\right)$ is a function representing the signal multiplexing relationship in the baseband.

$$
s(t)=\operatorname{Re}\left\{f\left(s_{i}(t), i=1, \ldots, N\right) e^{j 2 \pi f c t}\right\}
$$

where $\operatorname{Re}\{\}$ denotes a function that extracts the real part of the argument and fc is the carrier frequency.

In (2), the power variation of the signal $s(t)$ is directly related to the equivalent baseband signal component $f\left(s_{i}(t), i=1, \ldots, N\right)$ since the carrier signal cannot contribute the envelope variation of the signal. To make the power variation constant, the multiplexing function in the baseband should generate constant envelope baseband signal.

\footnotetext{
* Corresponding author: jmahn@ @nu.ac.kr
} 
The baseband multiplexing function serves to synthesize the service signals to be multiplexed. In this process, since the secondary code $\operatorname{Cs}(\mathrm{t})$, which is commonly applied to all service signals, is included, signal components other than $\mathrm{Cs}(\mathrm{t})$ are synthesized to generate a multiplexed signal. This synthesis process can be modelled as follows.

$$
f\left(s_{i}(t), i=1, \ldots, N\right)=g\left(s_{b, i}(t), i=1, \ldots, N\right) C_{s}(t)
$$

where, $g\left(s_{b, i}(t), i=1, \ldots, N\right)$ is a baseband multiplexing function for synthesizing multiplexed signal with binary service signals spread by primary code, and $s_{b, i}(t)$ represents an i-th binary service signal and can be expressed as $s_{b, i}(t)=d_{i}(t) C_{p, i}(t), i=1, \ldots, N$.

The power variation of the multiplexed signal can be obtained by taking the square of the absolute value of the result of equation (3) as follows

$$
\begin{aligned}
P(t) & =\left|f\left(s_{i}(t), i=1, \ldots, N\right)\right|^{2} \\
& =\left|g\left(s_{b, i}(t), i=1, \ldots, N\right)\right|^{2}\left|C_{s}(t)\right|^{2}
\end{aligned}
$$

where $\left|C_{s}(t)\right|^{2}$ is constant since the secondary code $C_{s}(t)$ is a complex valued function drawn unit circle in the complex plane. Therefore $\left|g\left(s_{b, i}(t), i=1, \ldots, N\right)\right|^{2}$ should be kept constant to make the multiplexed signal constant envelope.

Figure 1 shows the signal generator structure for generating multiplexed signal with constant envelope property where the signals for multiplexing are spread by TPC.

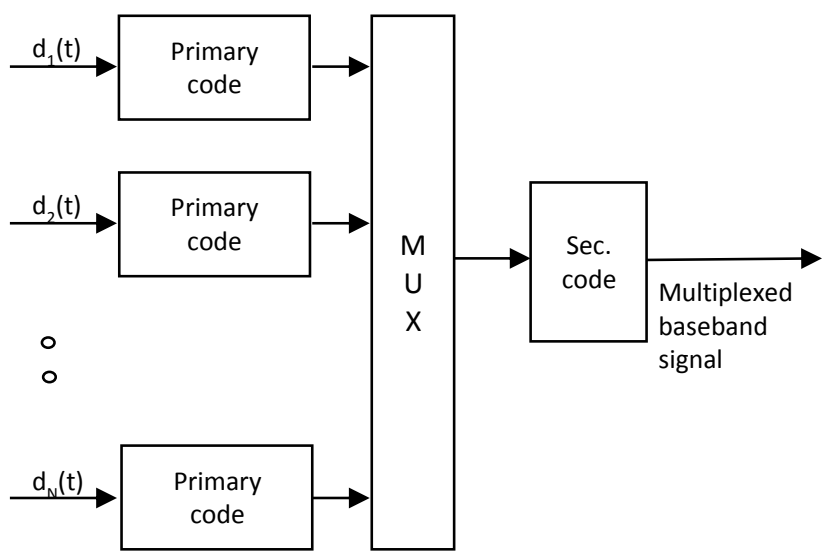

Fig. 1. Signal generator structure for TPC based signal multiplexing.

For the signal multiplexer in figure 1, there have been many research results achieving constant envelope characteristic for more than 3 binary signals multiplexing. By adding some intermodulation signals, they can achieve the constant envelope characteristics.

In this paper we add intermodulation terms and modify the gains of the signals to achieve the constant envelope characteristic of the multiplexed signal.

\section{Power efficiency and constellation}

The performance of the signal multiplexing can be measured by the power efficiency. The power efficiency is defined as the ratio of the sum of the signal powers at the multiplexed signal to the total signal power of the multiplexed signal. The total multiplexed signal power is the sum of the powers of signals and the powers in the intermodulation terms.

To evaluate the power efficiency of the multiplexed signal based on TPC spreading, we tried to find intermodulation terms and their weight. Also, to increase the power efficiency, we tried to modify the signal gains incorporated in the multiplexing process.

For a specific example, we tried to find a solution for the 3 signal multiplexing case. The baseline form of the multiplexed signal would be represented as follows

$$
s_{\text {mux }}(t)=s_{1}(t)+s_{2}(t)+j s_{3}(t)
$$

where $s_{i}(t), i=1,2,3$ is the signals for multiplexing and all of them are binary signals.

By numerical simulation, we have obtained the multiplexed signal form with constant envelope characteristic as follows

$$
\begin{aligned}
s_{\text {mux }}(t)= & 0.8 s_{1}(t)+0.8 s_{2}(t)+j s_{3}(t) \\
& -j 0.64 s_{1}(t) s_{2}(t) s_{3}(t)
\end{aligned}
$$

Figure 2 shows the constellation of the multiplexed signal with constant envelope property as shown in equation (6). By allowing the power allocation difference between signals for multiplexing up to $2 \mathrm{~dB}$, the power efficiency of the multiplexed signal in equation (7) becomes $84.77 \%$.

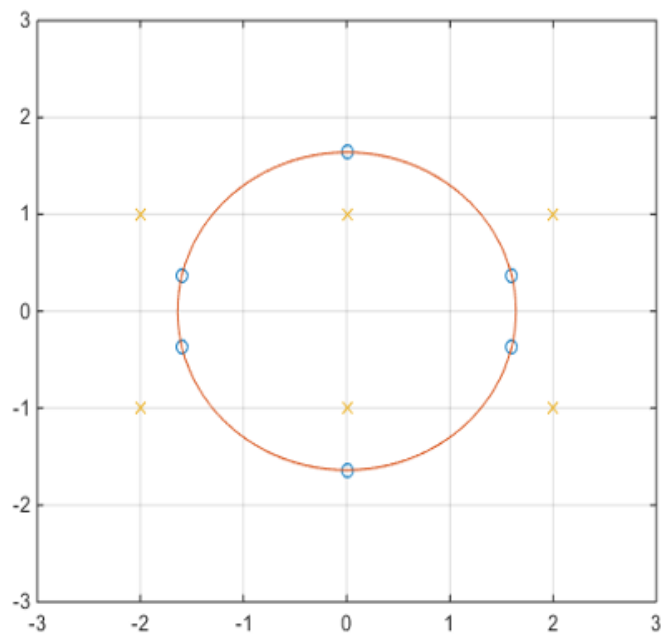

Fig. 2. Signal constellation of the multiplexed signal 
The results given in equation (7) and figure 2 illustrate that there may be many difference results by changing the maximum value of the power allocation difference.

\section{Conclusions}

In this paper, we proposed a signal generator structure for TPC based signal multiplexing. Due to the polyphase structure of the TPC, legacy binary signal multiplexing schemes could not be applicable to the problem such that the proposed reordering of the multiplexing and spreading works well in the TPC case.

The numerical simulation results for the proposed signal multiplexer show that the power efficiency of the proposed scheme for 3 signal multiplexing case approaches $85 \%$ and can be higher value with power allocation modification.

To verify the validity of the proposed scheme, more numerical results for 3 or more signals multiplexing.

This work has been supported by the National GNSS Research Center Program of the Defense Acquisition Program Administration and the Agency for Defense Development.

\section{References}

1. J. Kim, J. Lee, D. Park, K.J. Kim, K.W. Song, S.J. Lee, J.M. Ahn, IET Elec. Lett. 50, 218 (2014)

2. S.K. Han, J. Kim, J.K. Kim, A. Han, K.J. Kim, K.W. Kim, S.J. Lee, J.M. Ahn, IET Elec. Lett. 53, 598 (2017)

3. Z. Yao, M. Lu, IEEE Sig. Proc. Mag. 34, 16 (Sept. 2017) 